\title{
Virtual probes of mineral-water interfaces - the more flops, the better!
}

Andrew G. Stack ${ }^{1}$, Julian D. Gale ${ }^{2}$, and Paolo Raiteri ${ }^{2}$

1 Chemical Sciences Division, Oak Ridge National Laboratory

Oak Ridge, TN 37831, USA

E-mail: stackag@ornl.gov

2 Nanochemistry Research Institute and Department of Chemistry

Curtin University, GPO Box U1987, Perth WA 6845, Australia

E-mail: J.Gale@curtin.edu.au; P.Raiteri@curtin.edu.au

New approaches are allowing computer simulations to be compared quantitatively with experimental results, and they are also raising new questions about reactivity at mineralwater interfaces. Molecular simulations not only help us to understand experimental observations, they can also be used to test hypotheses about the properties of geochemical systems. These new approaches include rigorous calibration of simulation models against thermodynamic properties and atomic structure. They also encompass rare event theory methods that allow simulation of slow, complex mineral surface reactions. Here, we give an overview of how these techniques have been applied to simulate mineral-water interface structure, growth/dissolution mechanisms, and cluster formation.

KEYWORDS: simulation, molecular dynamics, calcite, barite, kinetics

\section{INTRODUCTION}

Experimental studies are increasingly revealing details of the nature of the mineral-water interface. Naturally, many scientists want to rationalize observations in terms of the fine detail of individual atomic interactions. However, making the connection between an experiment and individual atoms is a complex process. Here virtual experiments can be invaluable, since computer models can readily address the atomic scale and allow us to predict the observations that would result from a particular configuration of atoms (FIG. 1). By linking together theory, simulation, and experiment, it is increasingly possible to 
connect the macroscopic to the microscopic and ultimately to individual atomic positions and motions. Also, we are now moving away from an era when the role of simulations was merely to allow post hoc rationalization of experiment to one in which simulations can be a predictive tool in their own right. Here we illustrate how this can be achieved for two important examples, namely calcium carbonate $\left(\mathrm{CaCO}_{3}\right)$ in several forms and barite $\left(\mathrm{BaSO}_{4}\right)$. However, the implications are far broader and extend to the majority of mineral-water interfaces.

\section{WHAT IS MOLECULAR SIMULATION?}

For our purposes, the aim of a simulation is to make an accurate computer model of a geochemical system at the atomic level. Because the interface between a mineral and water involves a solid-liquid phase boundary, no single, unique atomic structure can be considered representative. Instead there exists a collection of many different molecular arrangements that are constantly fluctuating. Furthermore, this fluid will usually contain ions that are in a dynamic equilibrium involving isolated solvated species, ion pairs, surface adsorption sites, etc. Hence instead of making a calculation, where we might probe only the lowest energy state of the system, we must perform a simulation, in which we have to probe all accessible states and their average properties. The quality of a simulation critically depends on the ability to successfully visit the important states of a system and determine the probability with which they occur; we can then access the free energy landscape, which ultimately determines all of the chemical and physical properties.

Navigating this rugged landscape to make an accurate map of the free energy represents a significant challenge. Even addressing a sample containing some multiple of an Avogadro's number of distinct atoms, as in an experiment, is impossible with the computer. However, by taking a smaller volume - one containing typically between a few thousand and a million atoms - wrapped within a repeating unit to avoid surface effects, it is possible to mimic real systems, such as a step on a mineral surface or a nanoparticle in contact with a solution of ions. By studying many different mineral-water 
environments through separate simulations, a picture of the combined behavior of many possible scenarios can be reached.

How do we explore this world of possible atomic arrangements that are in constant motion? Here we will focus on the most popular approach, which is known as molecular dynamics (MD). Formally, this is a method where atoms move with time according to Newton's classical laws of motion based on the force exerted by other atoms; the method starts from an initial configuration and a set of velocities corresponding to the kinetic energy of the atoms at a desired temperature. Not only will the dynamics of the atomic motions be obtained, but after a sufficiently long simulation, the equilibrium structural and thermodynamic properties can also be determined. Informally, the method can be likened to skiers launching down a high mountain slope on frictionless ice; they will explore valleys and ridges happily until one of Newton's other scientific contributions, namely gravity, stops them.

Molecular dynamics can yield everything from thermodynamic properties to diffusion coefficients, but it is vital to be aware that there are limitations, especially in the context of mineral-water interfaces. The most significant limitation arises because MD is a numerical method that follows the time evolution of a system by taking small, discrete steps. The largest possible step size that limits the numerical error is determined by the fastest atomic motion in the system; for water, this is given by the stretching vibrations of the $\mathrm{O}-\mathrm{H}$ bond. Based on this, each time step is usually no more than 1 femtosecond $\left(10^{-15}\right.$ s). With modern computers, this makes it practical to study routinely up to $\sim 100$ nanoseconds $\left(1 \mathrm{~ns}=10^{-9} \mathrm{~s}\right)$ of real time. Consequently, molecular dynamics can only be used directly to study processes that occur on short timescales. To place this in context, a relevant timescale for mineral interfaces and solutions is the rate at which water that coordinates a dissolved metal cation exchanges, since all metal speciation reactions require this to occur. For $\mathrm{Ca}^{2+}{ }_{(a q)}$, the average lifetime of water molecules and other simple ligands around the ion at ambient conditions is known to be short, less than $10 \mathrm{~ns}$ (Eigen 1963). A simulation that lasts for $100 \mathrm{~ns}$ is therefore likely to reach equilibrium. In contrast, for $\mathrm{Mg}^{2+}$ (aq) the average lifetime of water is $\sim 53,000 \mathrm{~ns}$ (Richens 1997), and 
therefore a standard molecular dynamics simulation using today's computers cannot say anything meaningful regarding the speciation of such strongly hydrated ions, as it is improbable that even a single exchange will be observed during the entire simulation! While this may appear to be a major problem for MD, many tricks can be played to accelerate reactions (see below). While such methods can improve the exploration of the free energy surface, the downside is that the meaning of time is often lost, and so kinetic information can no longer be extracted just by using a stopwatch.

\section{HOW CAN WE COMPUTE THE INTERACTIONS BETWEEN ATOMS?}

The key input to a molecular dynamics simulation is a model for the interactions between atoms that will determine the forces and thus the energetics and dynamics. Ideally, these forces would come from very accurate quantum mechanical calculations; then, it just becomes a matter of sampling configurations until everything of interest is known to within acceptable statistical error. Fortunately life is not that easy, and this keeps skilled computational geochemists in employment!

Studying mineral-water interfaces using quantum mechanical techniques largely constrains the practitioner to use so-called density functional theory as the method best suited to condensed phases. Even using the computationally least expensive methods that explicitly include electrons, a simulation of a water-mineral interface is like burying a dead elephant: it's a major undertaking! Practical requirements typically restrict the system size to a few hundred atoms and simulated time to a few tens of picoseconds. This is often less than the time taken for a simulation to settle down, and so the interpretation of any statistical information requires care. Furthermore, current quantum mechanical methods suited to mineral-water systems exhibit systematic errors that can be important. For example, water densities are often too low and show incorrect diffusivities. Hence "tricks" are often used, including fixing the density at the experimental value and increasing the temperature by $20 \%$ beyond the temperature of interest (Wang et al. 2011). On the positive side, quantum mechanical methods do allow to examine a wide range of chemical reactivity, such as hydrolysis reactions. 
A pragmatic alternative to using computationally expensive, parameter-free methods is to take a more approximate approach. For example, one can dispense with the explicit treatment of electrons and employ a force field description that focuses only on empirical relationships between the forces and the atom positions. This reduction in complexity is compensated for by introducing parameters that are fitted to reproduce experimental information. The quality of the model thus depends on the approximations made and the data used to derive the parameters. The challenge of simulation is to find the best compromise between a realistic set of forces, a realistic description of the system size, and a timescale for the processes. Once this is done we are ready to apply simulation to real problems.

\section{PROBING STRUCTURE AT THE MINERAL-WATER INTERFACE}

Historically, determining the structure of mineral-water interfaces has been a challenge because of the need to conduct in situ experiments. With the advent of atomic force microscopy (AFM), it has become routinely possible to probe mineral surfaces as they grow and dissolve, thereby obtaining a wealth of information (Ruiz-Agudo and Putnis 2012). While incredibly valuable, AFM only provides part of the story since the solution and direct chemical information are invisible to the microscope. Grazing-incidence diffraction techniques are capable of resolving both the mineral structure and any ordered water layers above the interface. Its application has been limited somewhat by the requirement for relatively large, flat terrace regions.

The leading example of a mineral-water interface where experimentation and simulation have come together is the dominant surface of calcite $\left(\mathrm{CaCO}_{3}\right)$. Here, grazing-incidence X-ray experiments have identified the presence of two ordered near-surface layers of water, as well as the presence of distortions to the carbonate orientation that propagate several layers into the mineral (Geissbühler et al. 2004). Simulations also demonstrate the presence of ordered water at this surface, with the height of the first oxygen layer being 2-2.5 $\AA$ above the plane of the upper calcium ions, depending on the model used. The 
latest X-ray refinements give a value of $2.14 \AA$ for this distance, in agreement with simulations based on the most extensively calibrated force field for this system (Raiteri et al. 2010). Aside from quantitative comparison, the simulations provide information on water ordering, both parallel and perpendicular to the surface (FIG. 2). Here, the first layer of water is found to coordinate through oxygen to calcium, while the hydrogen of one $\mathrm{O}-\mathrm{H}$ group is bonded to carbonate such that the $\mathrm{O}-\mathrm{H}$ group is parallel to the surface plane. In the second layer of water, $\mathrm{O}-\mathrm{H}$ groups are perpendicular to the surface, thereby allowing hydrogen to bond to carbonate through the gaps in the first water layer.

Given the good agreement between simulation and experiment of the water structure above the calcite surface, it is possible to simulate other environments more difficult to access experimentally. An example of this is the hydration of amorphous calcium carbonate (ACC), which is known to have a variable amount of water within its structure. Recent analysis of experimental-pair distribution functions indicates the existence of two atomic arrangements that may be representative of the real disordered structure and suggests that water congregates in channel-like regions, rather than being homogeneously distributed (Goodwin et al. 2010). Here, simulation has been used to test how reasonable these models are (Singer et al. 2012). However, simulation can go further and probe the very formation of ACC from nanoparticles and how they are solvated (Raiteri and Gale 2010). Unlike calcite, with ACC there is no observed ordered water layer because the disordered mineral frustrates the ability of water to organize (see FIG. 2). This has important consequences for the early stages of $\mathrm{CaCO}_{3}$ growth at the nanoscale. Simulation predicts that ion pairs can add to ACC nanoparticles with little or no kinetic hindrance, while the ordered water layer on the surface of calcite initially acts to repel growth species. Hence, water ordering plays a vital role in controlling the rate of mineral growth from solution.

\section{THERMODYNAMICS OF MINERAL-WATER INTERFACES}


When a mineral surface is created in vacuo, the thermodynamic cost of this process is the surface energy. While calculated surface energies have been used extensively over the past decades to make predictions of preferred crystal shapes, it is more difficult to obtain the interfacial free energy between a mineral and bulk water, both experimentally and in simulation. However, the thermodynamics of ion attachment to or detachment from the mineral surface while immersed in water can be readily determined, provided a reaction coordinate can be defined that distinguishes between the ion at the surface and in solution. Often, the height above the surface is sufficient as a coordinate, assuming the dynamics of the water around the ion are fast relative to the simulation timescale.

Barite is an example where the free energy profiles for ion attachment are illuminating. This insoluble mineral is a common pipe scale in the oil and gas industries. Calculations of surface energies suggest that there should be 3 main surfaces for barite: (001), (010), and (210). The latter two surfaces would both be expected to be prominent morphologically, as they have similar energies. In reality, the (010) face is absent from crystals when grown at low supersaturation where nucleation is rate limiting. Examination of the free energies for the addition of ions to the surfaces reveals why this is the case (Piana et al. 2006) (FIG. 3). First, the addition of $\mathrm{Ba}^{2+}{ }_{(a q)}$ to the terraces is endothermic in all cases, as the cost of water loss from the cation is not compensated for by the surface binding; in contrast, sulfate shows some tendency to bind, indicating that barium adsorption is rate limiting. Second, when a sulfate ion is already bound to the surface, the addition of $\mathrm{Ba}^{2+}{ }_{(\mathrm{aq})}$ becomes exothermic because formation of the ion pair stabilizes the reaction. Third, and most significantly, the sulfate ion is able to assist in the removal of water from barium on the (010) surface and thereby reduces the barrier to nucleation. This kinetic effect is specific to the mineral-water interface for the (010) surface and leads to fast growth, causing this face to disappear since crystal morphologies are dominated by the slowest growing faces.

Ion addition to the surface of calcite has also been examined in several simulation studies (Kerisit and Parker 2004; Raiteri et al. 2010). Again, the qualitative findings are similar to those for barite, suggesting some general principles for the thermodynamics of mineral 
growth. In a binary system, the ion with the least exothermic free energy of hydration will attach to the surface first (assuming equal concentrations of the mineral's constituent ions in solution). For minerals like carbonates and sulfates that contain molecular anions, the negative species will show greater surface adsorption while the cations will show little or no tendency to bind on the terraces alone. It has also been observed that organic molecules, such as the (poly-)aspartate molecule, can accelerate the growth of both calcite (Elhadj et al. 2006) and barite (Piana et al. 2007). Here, simulation reveals that the multifunctional nature of the molecule allows it to anchor to the surface while helping to shuttle the cation from the solution to the surface. This goes against the conventional view that organic additives only poison the growth of minerals by blocking sites. Instead, it now appears that some molecules can accelerate growth, at least at low concentrations.

Before closing this section, which highlights a few simulation successes for mineralwater interfaces, some words of caution are in order. Since most simulations rely on force fields, it is vital to recognize that these studies are only as good as the data used to calibrate the model. To date, the majority of force fields have been fitted to structureproperty data of minerals in the solid state, but often, less consideration has been given to thermodynamic calibration, especially with respect to the interaction with water. For example, many recent simulations on aqueous calcium carbonate systems have been performed using models that have failings, including predicting aragonite to be more stable than calcite at ambient conditions, water that is freezing at room temperature, and calcite solubility that is in error by a factor of $10^{20}$ (Raiteri et al. 2010). Some of these failings are not just technical issues, but they can change the overall conclusions. For example, underestimating the free energy of solvation can lead to calcium sticking to the planar surfaces of calcite instead of being repelled. Given these issues, how can a budding computational geochemist judge the quality of a computer model? Well, here are a few tricky questions that you can ask your friendly theoretician:

- How accurate is the free energy of solvation of the ions in water?

- How accurate is the free energy of dissolution (or $K_{s p}$ ) value? 
- How accurate is the density of the water model, and does the water have the right mobility at the conditions of interest?

- Does the exchange rate of water at the cations agree with experiment?

- Does the model predict the correct ground-state mineral polymorph?

- How does the ion-pairing free energy compare with the experimental value?

If the answers are evasive, perhaps there are some issues with the force field model.

\section{REACTION SIMULATION FOR THE IMPATIENT}

Aside from structure and thermodynamics, we can also use atomistic simulations to understand the rates of reactions. We might try to run a molecular dynamics simulation and count the number of times a certain process occurs in order to obtain the rate. This works well for fast reactions, such as those that are limited by diffusion, for example, water movement in the interlayer of clays (Kalinichev et al. 2000) or water exchange at some mineral surfaces (Kerisit and Parker 2004), but many geochemical reactions are just too slow. We thus need ways to calculate the rates of these infrequent reactions, but by running the simulations for practical amounts of time.

One way around the above problem is to compute the free energy barrier (activation energy) for a reaction to occur and then try to relate this to the reaction rate. People have been using this approach, with small molecular fragments or mineral surfaces with a limited number of water molecules, for decades. Examples of this approach include the water exchange reactions of ions (Rotzinger 2005) and polyoxometalates (Stack et al. 2005), as well as dissolution (Wallace et al. 2010; Kubicki et al. 2012). However, the derived reaction mechanism and thermodynamics are often determined by the details of exactly how many water molecules are included and their structure (Evans et al. 2008). The challenge is therefore to go beyond looking for a single transition state in molecular calculations and instead find likely ensembles of reaction pathways within a dynamic simulation that includes bulk water. 
Fortunately many methods allow one to calculate the rates of processes that occur too infrequently to simulate directly. We will refer to these as "rare event theories." Central to such theories is the idea of bias, that is, forcing the simulation to spend time in states that might not normally be visited. This bias can take many forms, including, for example, a potential that constrains atoms to follow a predetermined pathway between two positions (umbrella sampling) or a force that makes molecules move away from the region they currently occupy (metadynamics). The results of the biased simulation can be transformed to provide the probability of visiting each state. In turn, this gives the free energy landscape, hopefully including the transition, reactant, and product states of the reaction of interest.

Once the magnitude of the activation energy is known, the rate constants can be estimated using transition state theory. By taking the ratio of the rate constants for the forward and reverse reactions, one can calculate the equilibrium constant. On its own, this method has most often been employed geochemically to examine ion-pairing reactions (Larentzos and Criscenti 2008). These are important to simulate because the equilibrium constant for ion-pair formation in thermodynamic databases is often inferred from an assumed activity-concentration relationship, such as the Debye-Hückel model, instead of being measured. Little is known about the actual ratios of solvent-separated versus contact ion pairs, except as determined using dielectric relaxation spectroscopy when applicable. Molecular simulation is helping to establish which ion pairs form and why.

One problem with rate constants determined simply from the free energy landscape is they are computed assuming that every crossing of the transition state leads to products. In reality, some reactants attempting to cross the transition state will go back to being reactants, and so the above rate constant has to be multiplied by the fraction of successful reaction attempts, that is, the transmission coefficient. Determining this is one of the most challenging tasks in simulation, though there is now an armory of methods to do this, such as the reactive flux technique (Chandler 1998). In this, one sets up a very large number $(1000+)$ of short simulations starting at the transition state and then measures the 
fraction in which the atoms reach either the reactant or the product without recrossing the barrier. The product of the transmission coefficient and the transition state theory rate constant is the overall rate constant, which should be directly comparable to experiment. However, rate "constants" measured for geochemical processes often represent a combination of different reactions. For example, a rate constant for growth or dissolution of a mineral is usually expressed as moles produced or consumed per unit volume per unit surface area of mineral, not in the units of an elementary reaction consisting of individual bond breakages or formations. In this case, one is forced to compare the virtual and experimental Arrhenius activation energies (Wang et al. 2007; Stack et al. 2012).

Geochemical examples where biased simulation and reactive flux methods have been combined successfully include water exchange reactions on aqueous ions (Stack and Rustad 2007), atomic clusters (Wang et al. 2007), and mineral surfaces (Kerisit and Parker 2004; Raiteri et al. 2010). Water exchange reactions in general are important because they are one of the processes that provide an upper bound for a reaction rate. In aqueous solution, a reaction cannot occur faster than the rate at which the reactants diffuse together and then exchange their coordinating water molecules prior to making bonds to something else. Often the water exchange rate correlates with the rate of dissolution of minerals with the same structure (Casey and Ludwig 1995), or with the effect a background electrolyte or impurity has on a reaction rate (Dove 1999). As an important point of validation, these studies have shown that, given a suitably accurate force field, molecular dynamics simulations can give rate constants that are within experimental uncertainty for some water exchange reactions (Stack and Rustad 2007; Kerisit and Rosso 2009).

In the above examples, the reaction pathway was already known. However, for many mechanisms this is not the case (for example, for mineral growth and dissolution, multiple bonds form or break and their ordering is not often known). Without this knowledge, it is hard to locate the correct pathway and obtain the reaction rate. One solution is to use metadynamics (Laio and Parrinello 2002), a technique in which the free energy landscape is explored with less a priori specification of the mechanism. This is 
achieved by requiring the system to gradually explore states that have not previously been visited. This approach was used recently by Liu et al. (2011) to measure detachment/attachment of ions from a halite $(\mathrm{NaCl})$ step edge, while Stack et al. (2012) used a combination of all the rare event theories previously mentioned to calculate rate and equilibrium constants for barium attachment/detachment to a barite step edge (FIG. 4). Both studies highlight the importance of an ion directly, but singly-bonded to the step edge as an intermediate state whose formation/destruction controls the rate of growth and dissolution. Furthermore, in Stack et al. (2012), the activation energies for the ratelimiting steps quantitatively matched those measured by AFM during growth and dissolution, an important validation of rare event theories.

\section{CONCLUSIONS}

We have shown some of the insights that can be gained by virtually probing mineralwater interfaces, including structural details, thermodynamics, and rate constants. We hope that these few examples suffice to show that atomistic computational simulation of geochemical processes has a bright future. The "tool kit" of virtual methods that geochemists have at their disposal to discover reaction mechanisms is powerful and becoming increasingly quantitative. By combining the detailed, simulated, atomic-scale kinetics with methods that work at longer length- and timescales it is possible to make macroscopic predictions. For example, the use of the kinetic Monte Carlo method is capable of reproducing the growth of molecular crystalline systems on the micron/millisecond scale (Piana et al. 2005). For mineral systems, this has not been possible until now due to the lack of rate constants for each ion addition or loss. However, the advent of rare event theories can now provide this missing kinetic information. With the relentless advance of computing power, as measured by the increasing number of floating point operations per second (i.e., flops), the next decade will see the prediction of mineral growth from an aqueous solution based on atomistic simulations change from vision to reality. 


\section{ACKNOWLEDGMENTS}

This research was sponsored by the Division of Chemical Sciences, Geosciences, and Biosciences, Office of Basic Energy Sciences, U.S. Department of Energy (AGS) and the Australian Research Council through Discovery Grant DP0986999 (PR and JDG).

\section{REFERENCES}

Casey WH, Ludwig C (1995) Silicate mineral dissolution as a ligand-exchange reaction. Reviews in Mineralogy 31: 87-118

Chandler D (1998) Finding transition pathways. In: Berne BJ, Ciccotti G, Coker DF (eds) Classical and Quantum Dynamics in Condensed Phase Simulations. World Scientific, Singapore, pp 51-66

Dove PM (1999) The dissolution kinetics of quartz in aqueous mixed cation solutions. Geochimica and Cosmochimica Acta 63: 3715-3727

Eigen M (1963) Fast elementary steps in chemical reaction mechanisms. Pure and Applied Chemistry 6: 97-115

Elhadj S, De Yoreo JJ, Hoyer JR, Dove PM (2006) Role of molecular charge and hydrophilicity in regulating the kinetics of crystal growth. Proceedings of the National Academy of Sciences 103: 19237-19242

Evans RJ, Rustad JR, Casey WH (2008) Calculating geochemical reaction pathways Exploration of the inner-sphere water exchange mechanism in $\mathrm{Al}\left(\mathrm{H}_{2} \mathrm{O}\right)_{6}{ }^{3+}(\mathrm{aq})+n \mathrm{H}_{2} \mathrm{O}$ with ab initio calculations and molecular dynamics. Journal of Physical Chemistry A 112: $4125-4140$

Geissbühler P, Fenter P, DiMasi E, Srajer G, Sorensen LB, Sturchio NC (2004) Threedimensional structure of the calcite-water interface. Surface Science 573: 191-203

Goodwin AL, Michel FM, Phillips BL, Keen DA, Dove MT, and Reeder RJ (2010) Nanoporous Structure and Medium-Range Order in Synthetic Amorphous Calcium Carbonate. Chemistry of Materials 22: 3197-3205

Kalinichev AG, Kirkpatrick RJ, Cygan RT (2000) Molecular modeling of the structure and dynamics of the interlayer and surface species of mixed-metal layered hydroxides: 
Chloride and water in hydrocalumite (Friedel's salt). American Mineralogist 85: 10461052

Kerisit S, Parker SC (2004) Free energy of adsorption of water and metal ions on the 110 14 \} calcite surface. Journal of the American Chemical Society 126: 10152-10161

Kerisit S, Rosso KM (2009) Transition path sampling of water exchange rates and mechanisms around aqueous ions. Journal of Chemical Physics 131:114512 (15 pp)

Kubicki JD, Sofo JO, Skelton AA, Bandura AV (2012) A new hypothesis for the dissolution mechanism of silicates. Journal of Physical Chemistry C 116: 1747917491

Laio A, Parrinello M (2002) Escaping free-energy minima. Proceedings of the National Academy of Sciences 99: 12562-12566

Larentzos JP, Criscenti LJ (2008) A molecular dynamics study of alkaline earth metalchloride complexation in aqueous solution. Journal of Physical Chemistry B 112: 14243-14250

Liu L-M, Laio A, Michaelides A (2011) Initial stages of salt crystal dissolution determined with ab initio molecular dynamics. Physical Chemistry Chemical Physics 13: $13162-13166$

Piana S, Reyhani M, Gale JD (2005) Simulating micrometer-scale crystal growth from solution. Nature 438: 70-73

Piana S, Jones F, Gale JD (2006) Assisted desolvation as a key kinetic step for crystal growth. Journal of the American Chemical Society 128: 13568-13574

Piana S, Jones F, Gale JD (2007) Aspartic acid as a crystal growth catalyst. CrystEngComm 9: 1187-1191

Raiteri P, Gale JD (2010) Water is the key to nonclassical nucleation of amorphous calcium carbonate. Journal of the American Chemical Society 132: 17623-17634

Raiteri P, Gale JD, Quigley D, Rodger PM (2010) Derivation of an accurate force-field for simulating the growth of calcium carbonate from aqueous solution: A new model for the calcite-water interface, Journal of Physical Chemistry C 114: 5997-6010 Richens DT (1997) The Chemistry of Aqua Ions. Wiley \& Sons, New York 
Rotzinger FP (2005) Treatment of substitution and rearrangement mechanisms of transition metal complexes with quantum chemical methods. Chemical Reviews 105: 2003-2038

Ruiz-Agudo E, Putnis CV (2012) Direct observations of mineral-fluid reactions using atomic force microscopy: the specific example of calcite. Mineralogical Magazine 76: $227-253$

Şimşek S, Yıldırım N, Gülgör A (2005) Developmental and environmental effects of the Kizldere geothermal power project, Turkey. Geothermics 34: 234-251

Singer JW, Yazaydin AÖ, Kirkpatrick RJ, and Bowers GM (2012) Structure and Transformation of Amorphous Calcium Carbonate: A Solid-State ${ }^{43} \mathrm{Ca}$ NMR and Computational Molecular Dynamics Investigation. Chemistry of Materials 24: 18281836

Stack AG, Rustad JR (2007) Structure and dynamics of water on aqueous barium ion and the $\{001\}$ barite surface. Journal of Physical Chemistry C 111:16387-16391

Stack AG, Rustad JR, Casey WH (2005) Modeling water exchange on an aluminum polyoxocation. Journal of Physical Chemistry B 109:23771-23775

Stack AG, Raiteri P, Gale JD (2012) Accurate rates of the complex mechanisms for growth and dissolution of minerals using a combination of rare-event theories. Journal of the American Chemical Society 134: 11-14

Wallace AF, Gibbs GV, Dove PM (2010) Influence of ion-associated water on the hydrolysis of Si-O bonded interactions. Journal of Physical Chemistry A 114: 25342542

Wang J, Rustad JR, Casey WH (2007) Calculation of water-exchange rates on aqueous polynuclear clusters and at oxide-water interfaces. Inorganic Chemistry 46: 29622964

Wang J, Román-Pérez G, Soler JM, Artacho E, Fernández-Serra M-V (2011) Density, structure, and dynamics of water: The effect of van der Waals interactions. Journal of Chemical Physics 134: 024516 


\section{FIGURES}

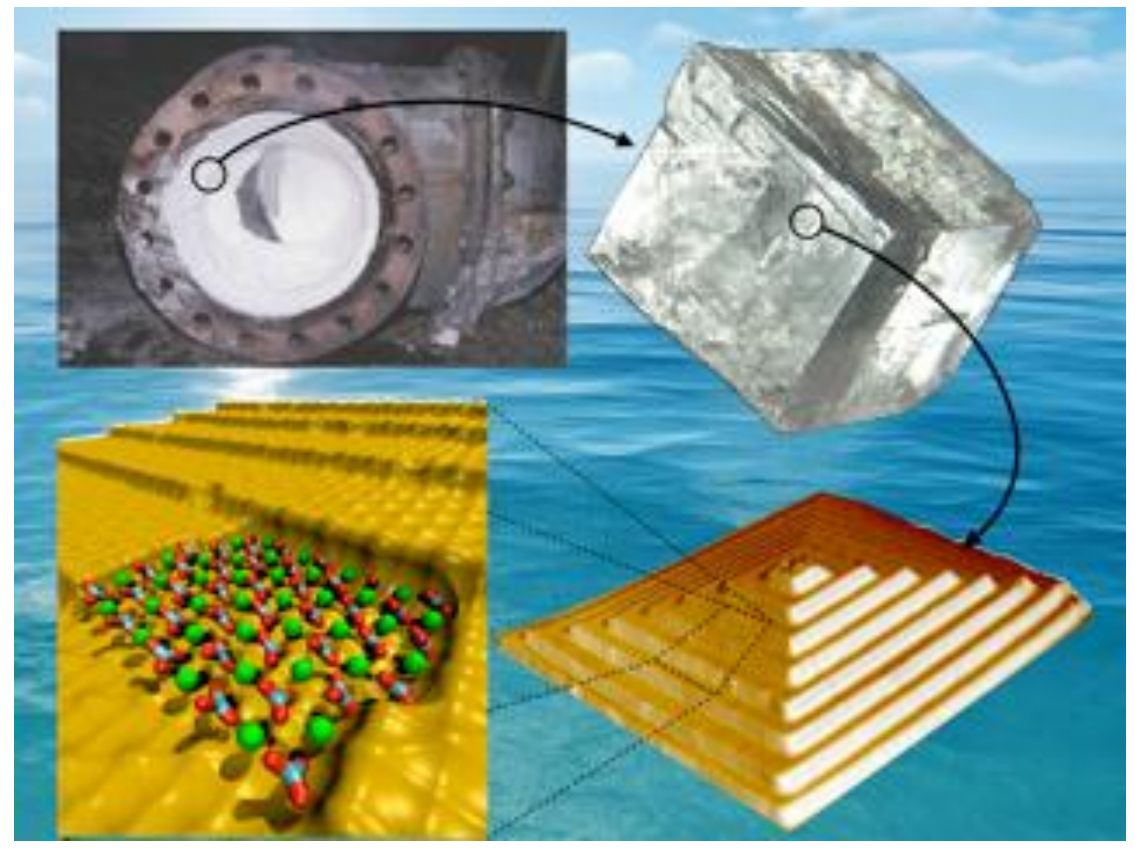

Figure 1 Growth of minerals over varying length scales. The formation of calcium carbonate scale in a pipeline (A) occurs through the agglomeration of many rhombohedral calcite crystallites (B). Each surface of a crystal grows by attachment of ions to monomolecular steps originating from screw dislocations $(\mathbf{C})$. How the individual ions add to each step depends on the atomic structure (D). Simulation can now compute the rate constants at this most detailed level, and these can be used to connect with microscopic and macroscopic observations. (A) FROM SIMSEK ET AL. (2005), REPRINTED WITH PERMISSION OF ELSEVIER 


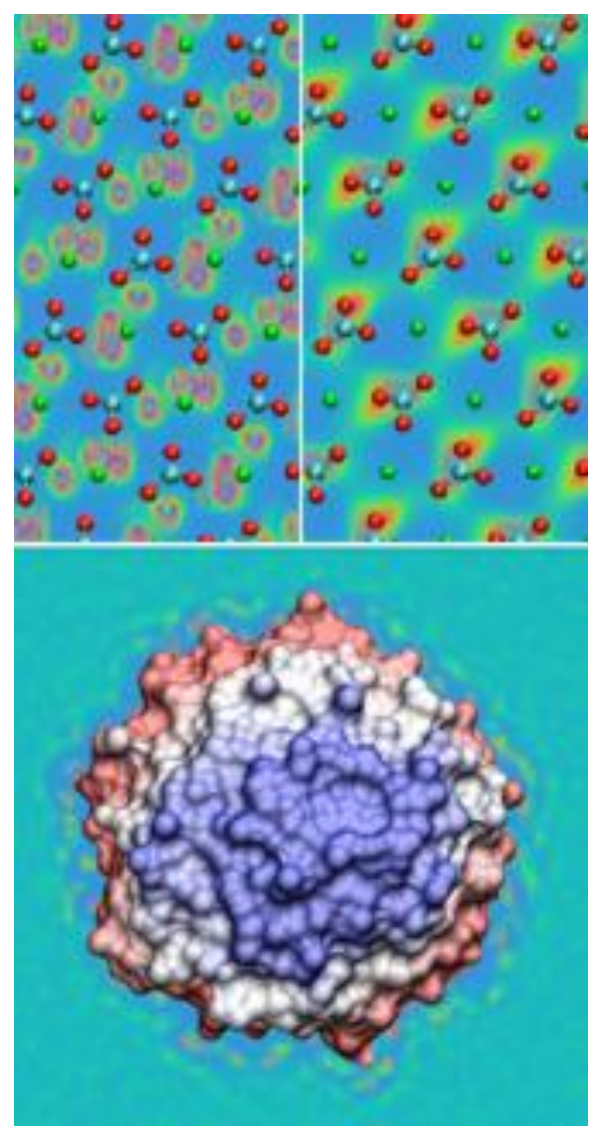

Figure 2 The influence of ordered water at mineral surfaces. X-ray diffraction has shown that there are two ordered layers of water above the surface of calcite (Geissbühler et al. 2004). Simulation can reveal how each layer orders parallel to the plane of cleavage (first layer above the surface, top left; second layer, top right). In the illustrations, color variations in the background from blue to red represent low to high density of water; the calcite atomic structure is superimposed for reference (here atoms are shown as spheres with $\mathrm{Ca}, \mathrm{C}$ and $\mathrm{O}$ being colored green, blue and red, respectively). In contrast, nanoparticles of amorphous calcium carbonate (lower panel) show less ordering of the water (shown for a plane that passes through the center) as the disorder of the surface frustrates the solvent. Note that the surface of the nanoparticle is colored by depth for a 3D perspective. 


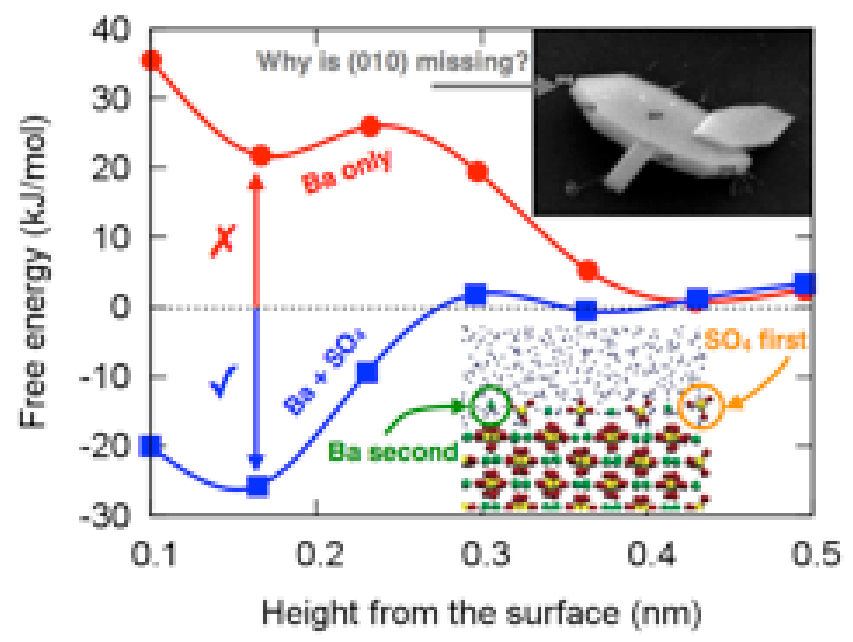

Figure 3 Free energy profiles illustrating why the (010) surface of barite $\left(\mathrm{BaSO}_{4}\right)$ is "missing." When barium ions try to bind to this surface on their own (red curve) the free energy goes up, whereas when they bind next to sulfate, stable adsorption occurs (blue curve). The lack of a barrier on the blue curve leads to rapid nucleation of new layers of $\mathrm{BaSO}_{4}$ (see inset) and overall fast kinetics, so that the (010) surface grows out. Adapted from Piana et al. (2006), with permission of the American Chemical Society 


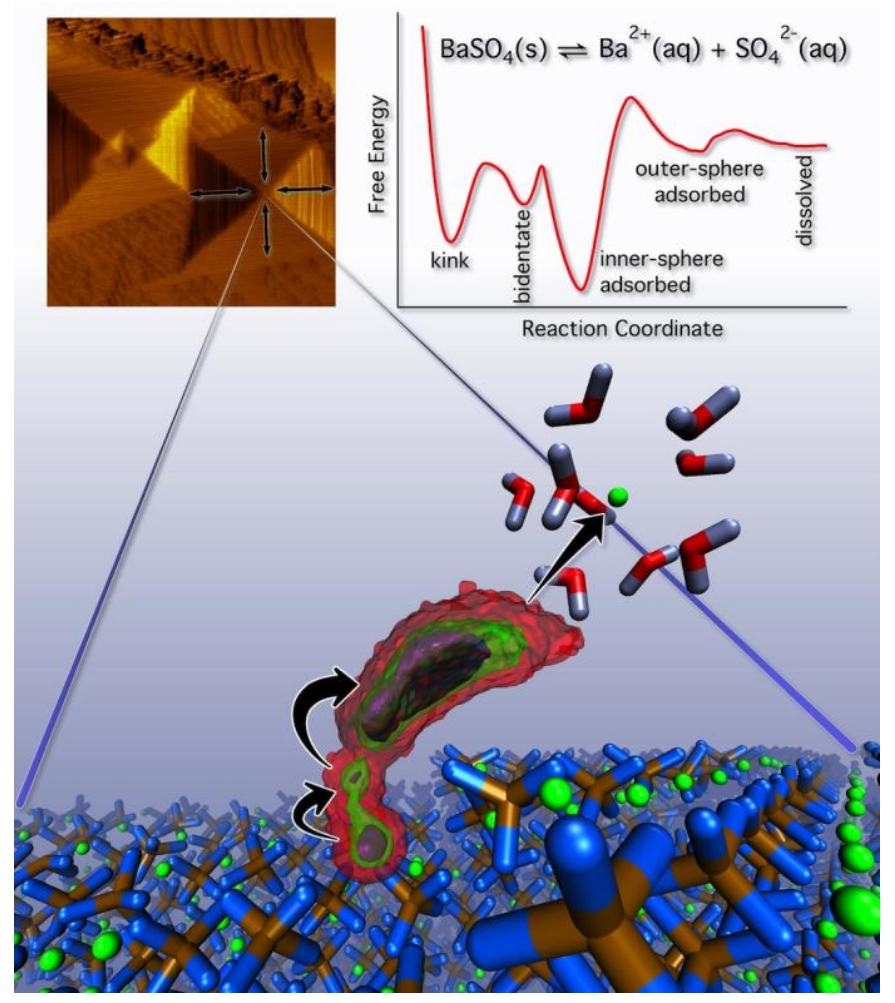

Figure 4 Rare event theory calculations. Metadynamics was used to discover the reaction mechanism (bottom) for barium-ion detachment from a step edge. The colored areas are the free energy landscape (low to high energy follows purple, green, red), which was quantified using umbrella sampling and reactive flux (top right). The activation energies for the rate-limiting steps for attachment to and detachment from the steps match those measured using AFM (top left). FROM STACK ET AL. (2012), WITH PERMISSION OF THE AMERICAN CHEMICAL SOCIETY 